Volume and Issues Obtainable at Center for Sustainability Research and Consultancy

Journal of Accounting and Finance in Emerging Economies

ISSN: 2519-0318\&ISSN (E):2518-8488

Volume 7: Issue 3 September 2021

Journal homepage: www.publishing.globalcsrc.org/jafee

\title{
Corporate Governance Performance Relationship and the Role of Institutions and Culture: New Evidence from Asian Microfinance Institutions
}

\begin{abstract}
Ahmad Nawaz, Department of Economics, COMSATS University Islamabad, Lahore Campus, Pakistan

Sidra Shahbaz, COMSATS University Islamabad, Lahore Campus, Pakistan

Abdul Farooq, Department of Economics, COMSATS University Islamabad, Lahore Campus, Pakistan

*Muhammad Masood Anwar, Women University of Azad Jammu Kashmir, Bagh, Pakistan

*Corresponding author's email: smasood_ajk@yahoo.com
\end{abstract}

\begin{tabular}{l}
\hline ARTICLEDETAILS \\
\hline History \\
Revised format: Aug 2021 \\
Available Online: Sep 2021
\end{tabular}

Keywords

Microfinance, Corporate

Governance, Performance,

Culture

\section{JEL Classification}

G20, G21

\section{ABSTRACT}

Purpose: In a globalized world today, Microfinance Institutions (MFIs) are concerned about their corporate governance mechanism to enhance financial and social performance. However, it largely depends on the existing institutional, cultural and economic factors. This paper furthers the debate on the impact of corporate governance on the financial and social performance of Microfinance Institutions (MFIs) in Asian Context.

Design/Methodology/Approach: The paper utilizes a panel cross-country data set comprised of 183 MFIs in 18 Asian countries over the period of 2010-2018. For empirical analysis, it applies GMM regression technique to control for the endogeniety issue.

Findings: The results show that generally corporate governance mechanism contributes more towards social performance of MFIs than the financial performance and a conducive institutional environment enhances both financial and social performance. However, good cultural and economic values contribute only towards the social performance of MFIs.

Implications/Originality/Value: Since majority of MFIs irrespective of their status are socially oriented. Therefore, good corporate governance mechanism is more effective in enhancing social performance in particular. Progress towards human development contributes to both financial and social performance of MFIs.

(C)2021, The authors, under a Creative Commons AttributionNonCommercial 4.0

Recommended citation: Nawaz, A., Shahbaz, S., Farooq, A. and Anwar, M. M. (2021). Corporate Governance Performance Relationship and the Role of Institutions and Culture: New Evidence from Asian Microfinance Institutions. Journal of Accounting and Finance in Emerging 
Economies, 7 (3), 537-551.

\section{Introduction}

Microfinance institutions (MFIs) provide both financial and non-financial services to Relatively marginalized section of the society (Mersland and strom 2013; World Bank 2006). Corporate governance (CG) plays a very important role for structuring and protecting the objective of the organizations and help to achieve these objectives. "It is a process through which stakeholders guide the MFI to define and protect the mission and the institutions assets" Campion et al. (2008). The fact that corporate governance mechanisms affect firm's performance has been substantiated by ample literature (Zagorchev et al. 2015; Bozec et al. 2012; Fooladi et al. 2011; De Jong et al. 2002; Mitton 2002).In the microfinance context, recently CG performance relationship is empirically investigated in many studies (Nawaz et al., 2018; Thrikawalaet al. 2013; Galema et al. 2012; Hartarska and mersland, 2012; Tehuigoua 2010; Bassem 2009; Manderlier et al. 2009; Mersland and Strom, 2008; 2009; Coleman and Osei 2008; Polanco 2005).

However, the literature suggests that corporate governance-performance nexus is affected by various country specific economic, cultural and institutional factors which are investigated in few studies in the context of conventional firms (Strese et al. 2016; Li, J et al. 2016; Griffin et al. 2015; Handley et al. 2015). The controversy surrounds about the role of country level factors, whether they as external governance mechanisms which constrain managerial discretion within firms (e.g., investor protection, disclosure regimes, market for corporate control) or they should capture the national context and the outcomes of the quality of national governance systems. Kaufmann et al. (2011) argues that governance research would benefit from finer distinction between rules-based and outcome-based indicators of national governance. Whereas rules-based indicators measure if countries have adequate anticorruption legislation or agencies and outcome-based indicators capture if anticorruption laws are actually enforced. Schiehll and Martins (2016) highlights the contextual factors to distinguish between the country and firm specific governance mechanisms. Which act as external mechanisms by reducing managerial through highlighting the agency conflicts within the firm. Besides cultural effects vary across countries in ethical context because of the difference in sensitivity (Simga-Mugan et al. 2005), value system (Ford et al. 2005), judgments (Whipple and Swords 1992), decision making (Srnka 2004), and perception (Vitell and Paolillo 2004) among others. Therefore, the corporate governance-performance relationship at cross country level controlling for institutional, cultural and economic factors is an area worth investigating.

In this backdrop, this paper aims to find out "to what extent cultural, institutional and economic factor affect the relationship between corporate governance-performance relationship. And more specifically "Do cultural, institutional and economic factors affect the relationship between corporate governance and MFIs financial and social performance in Asia?"

The paper is organized as follows. Section II discusses the relevant literature followed by research methodology presented in section III. The descriptive and empirical analysis is presented in section IV. Finally, we conclude in section V.

\section{Literature Review}

The essence of corporate governance is to enhance the welfare gains in a society and one way of achieving this is to increase the performance of firms (Mersland and Strøm 2010; Labie 2001). However, considering the agency theory, suggesting a conflict between the managers 
and owners, a permanent conflict exists between managers and owners, the corporate governance practices comes handy in minimizing these conflicts through various internal and external corporate controls, competition in the market, regulations regulating the institutional structures and financial markets (Jensen, 1993). The literature provides conclusive evidence of the effectiveness of good CG controls in increasing the firms performance (Liu et al, 2015; Black and Kim, 2012) which eventually leads to decision making strategically devised to increase the firms revenues through strict monitoring and controls (Salim et al. 2016; Rock et al. 1998). The optimum value of board size is one of many other effective control mechanisms, for which the literature provides ample evidence. As suggested by Jensen (1993), the optimal number of Bods (Board of Directors) is around seven or eight members, beyond which the performance of board starts diminishing. Whereas the Lipton and Lorsch (1992) suggested this optimal number to be ten members, beyond which it is difficult to summarize the opinions of all members. In social performance perspective, Hartarska and Mersland (2012) finds this optimal board size to be of nine members.

Along with the board size, the literature highlights other effective corporate control mechanisms which are the presence of international directors on board (Herrera et al. 2016; Beislandet al. 2014;Mersland et al. 2009) qualification of board members (Manderlier et al. 2009), and gender diversity (Thrikawala et al. 2013; Bassem 2009). Chen (2015) finds a strong relationship between board structure and firm characteristics. Board diversity affects the decision-making processes (Varhegyi, 2016; Rao \&Tilt 2015). Female representatives on board leads to increased firm performance (Tremblay 2016; Thrikawala et al. 2013; Bassem 2009). MFIs can generate incentives and mechanisms to improve their social performance by intentionally choosing board members with more social oriented profiles (Mori et al. 2015). The experience and knowledge of board member in the field of microfinance also increase efficiency (Manderlier et al.2009). Another corporate governance indicator which is highly used in literature is CEO duality (Duru et al. 2016; Tang 2016; Van Damme et al. 2016; Walthoff-Borm 2015; Beisland et al. 2014 Thrikawala et al., 2013). Agency theory advocates that in firm having separate CEO \& chairman, the conflict between management and board tends to be minimized which increases firm performance (Coleman and Osei.2008). On the other hand, once the role of CEO and chairman of the board is combined, the CEO is likely to have greater control and liberty in decision making which leads to riskier decisions (Galema et al. 2012; Hartarska et al. 2012; Mersland et al. 2009). MFIs performance is also affected by its ownership status. Nonprofit organizations (NPOs) focus more on the social welfare of the society while for-profit shareholder firms are more profit oriented (Gebremichael et al.2016; Salustri, et al.2015). NPOs are more prone to agency conflicts as compared to SHFs, because of their weak governance and monitoring structure (Galema et al. 2012). Regulation in SHFs, in addition to decreased agency conflicts, also provide some indirect benefits which ultimately increase the outreach of MFIs (Tchuigoua 2010; Lauer 2008; Mersland et al, 2008; 2009; Hartarska and Nadolnyak, 2007).Majority of NPOs, on the other hand, lend primarily to women particularly in Asia. Presence of female CEO in such MFIs contributes towards social performance (Strom et al. 2016; Périlleux et al. 2015;RØ Strøm et al. 2014; Mersland and strom 2009; Welbourne,1999). At cross-country level, the heterogeneity exists in cultural, institutional and economic conditions cause variation in corporate governance mechanism and its relationship with firm's performance (Hahn and Kühnen, 2013; Baughn et al. 2007; Welford, 2004, 2005; Maignan and Ralston, 2002; Howell, 2001). The absence of religious and military leaders from politics, lower levels of religious and ethnic tensions, and minimal levels of armed conflict are also related to institutional stability (Ramady, 2014). Chen and Hao (2011) argue that institutional institutions can affect the country's information environment and thus shareholders' ability to monitor firms. Similarly, Piotroski 
et al. (2010) claim that the benefits of transparency in many developing countries are masked by high levels of ownership concentration, politicized institutions, and corruption. Inclusive institutions contribute towards overall economic development of countries and in this process firms too are better regulated leading towards better performance (e.g., Roe and Siegel, 2009, 2011; Keefer, 2008; Rajan and Zingales, 2003). Glaeser et al. (2004) and Qi et al. (2010) argue that institutional institutions should reflect the ex-ante view of restrictions on government behavior rather than the ex post government performance. Culture also affects the relationship of corporate governance and performance. "It consists of the unwritten rules of the social game and is the mutual programming of the mind that differentiates the members of one group or category of people from others" (Hofstede et al., 2010). Heterogeneous societies divided among the cultural lines have greater element of mistrust among the society member unlike homogeneous societies (Strese et al, 2016; Li et al 2016; Engelen et al. 2014; Malik \& Zhao, 2013; Trompenaars \& Hampden1998; Sales \&Mirvis, 1984;). No wonders that prevailing national culture strongly effects the corporate governance and eventually firm's performance (Schneider \& Meyer, 1991; Langlois \& Schlegelmilch, 1990;). Cross- country evidence shows that there exists minimum connection between firm-level corporate governance practices and firm value in individualism societies. However, societies which avoid uncertainties have fond to have strong relationship between firm level corporate governance and performance (Dale Griffin et al., 2015).

\section{Methodology \\ Data and Sample}

Data from 183 MFIs in 18 Asian countries has been collected for the period of nine years from 2010 to 2018. Only 4 diamond rated and above MFIs listed on the mix market website are included in the sample. Overall the population comprised of 1684 MFIs located in 18 countries of Asia. The final sample included in the study is 183 MFIs having 4 diamond rating. Data for the financial and social performance indicators is taken from the MIX market website. Whereas, data related to the construction of CGI index is collected from various sources including rating reports of various agencies. The summary statistics of all independent and dependent variables and their definitions are presented in Table 3.1. The maximum value of CGI is 7 and the lowest is 2 . The average values of ROA and OSS are positive and majority of MFIs lend to women.

Table 3.1. Summary Statistics of the variables

\begin{tabular}{|c|c|c|c|c|c|c|}
\hline Variables & Definition & Mean & Std.Dev & Min & Max & Median \\
\hline 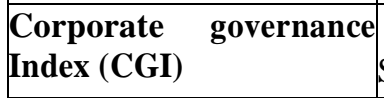 & Self-constructed at shown in table 3.2 & 4.062 & 1.028 & 2 & 7 & 4.09 \\
\hline $\begin{array}{lll}\text { Return on asset }(\%) \\
\left(\begin{array}{ll}\text { ROA) } \\
\text { ROAA) }\end{array}\right. & & \\
\end{array}$ & $\begin{array}{l}\text { net income after taxes and before } \\
\text { donations/average assets }\end{array}$ & 0.082 & 0.533 & -2.78 & 7.6 & 0.03 \\
\hline $\begin{array}{|ll|}\text { Operational se } & \text { If }-\mathrm{f} \\
\text { Sufficiency }(\%) & (\text { OSS })\end{array}$ & $\begin{array}{l}\text { financial revenues / } \\
\text { (financial expenses + loan Expenses } \\
\text { loan loss expenses }+ \\
\text { operating expenses) }\end{array}$ & 1.166 & 0.468 & -0.12 & 11.4 & 1.15 \\
\hline \begin{tabular}{|l|l|} 
Women to total \\
borrowers $(\%)$ \\
(WTB)
\end{tabular} & Percentage of women clients/ total clients & 0.709 & 0.308 & 0 & 1.1 & 0.81 \\
\hline Average loan size (\%) & Outstanding loans/ no. of borrowers & 0.386 & 0.596 & -0.78 & 6.13 & 0.19 \\
\hline $\begin{array}{l}\text { Human development } \\
\text { index (HDI) }\end{array}$ & $\begin{array}{l}\text { Three dimensions: Income, Health and } \\
\text { Education }\end{array}$ & 0.621 & 0.095 & 0.44 & 0.91 & 0.607 \\
\hline Domestic credit (DC) & Ratio of Domestic credit to private sector & 41.692 & 17.363 & 13.18 & 99.93 & 41.79 \\
\hline Globalization index (GI) $]^{K}$ & KOF index of Globalization & 52.232 & 7.885 & 34.5 & 75.51 & 51.36 \\
\hline
\end{tabular}




\begin{tabular}{|l|l|l|l|l|l|l|}
\hline Inflation (\%) & Taken from Worldbank data set & 7.629 & 8.412 & -0.94 & 247 & 6.91 \\
\hline Democracy index (DI) & Taken from the Polity IV data set & 0.338 & 1.190 & 0 & 5 & \\
\hline $\begin{array}{l}\text { Corruption perception } \\
\text { index (CPI) }\end{array}$ & Taken from Worldbank governance index & 30.005 & 7.462 & 18 & 53 & 28 \\
\hline Hofstede index (HI) & $\begin{array}{l}\text { Taken from Hofstede Index website } \\
\text { https://geert- hofstede.com/countries.html }\end{array}$ & 46.738 & 5.969 & 35 & 61 & 47.67 \\
\hline
\end{tabular}

\section{Corporate Governance Index}

This paper has extended the corporate governance index originally devised by Nawaz et al (2018). The selection of variables in the construction of CGI index is based on the existing literature among which, the board size (Salim et al; 2016: Jensen .1993), female CEO (Périlleux et al.2015; Mersland and strom, 2009; Strom et al.2016), board qualification (Manderlier et al. 2009), international directors (Mersland et al. 2009), CEO/Chairman duality (Thrikawala et al. 2013; Duru et al. 2016; Tang, 2016), and ownership type (Salustri, et al.2015).are extensively used

Table 3.2: Description of corporate governance Index

\begin{tabular}{|l|l|}
\hline Indicator & Description \\
\hline Board size & $\begin{array}{l}\text { Dummy equals 1 if the board size is greater than equals to } \\
\text { mean value and less than equals to 9, 0 otherwise. }\end{array}$ \\
\hline Female directors & $\begin{array}{l}\text { Dummy with value 1 if MFI has female presence in board, 0 } \\
\text { otherwise. }\end{array}$ \\
\hline Board qualification & $\begin{array}{l}\text { Dummy with value equals 1 if board has enough experience } \\
\text { and knowledge in microfinance, 0 otherwise. }\end{array}$ \\
\hline International & $\begin{array}{l}\text { Dummy with value equals } 1 \text { if board has no international } \\
\text { director, 0 otherwise. }\end{array}$ \\
\hline $\begin{array}{l}\text { CEO/Chairman } \\
\text { duality }\end{array}$ & $\begin{array}{l}\text { Dummy with value equals 1 if CEO and chairman roles are } \\
\text { separated, 0 otherwise. }\end{array}$ \\
\hline Ownership type & Dummy with value 1 if MFI is a SHF, 0 otherwise. \\
\hline Female CEO & $\begin{array}{l}\text { Dummy indicating value 1 if MFIs CEO is female, otherwise } \\
0 .\end{array}$ \\
\hline
\end{tabular}

Source: Nawaz et. al (2018)

\section{The Method}

\section{Dynamic Panel Generalised Method of Moment (GMM) Estimation}

In the conventional finance literature, the relationship between CG and firm performance has been thoroughly investigated. However, there exist potential sources of endogeneity leading towards biased results and only few recent studies have taken up this issue. However due to the complexity in identifying the exogenous factor in corporate governance, the researchers followed different approached to tackle the endogenity in this context fixed and random approach and conventional instrumental approach lack dealing with dynamic endogeneity which arises when the CG-firm performance relationship is influenced by past performance. The dynamic nature of this relationship is hardly been investigated in microfinance context (Mersland \& Strøm, 2009;Bassem, 2009; Hartarska, 2005;). As proposed by (Thrikawala et al.2017) this paper applies the dynamic panel generalized method of moments (GMM) technique to provide robust results. 
Based on GMM technique originally proposed by Hansen (1982) various studies (HoltzEakin, Newey, and Rosen (1988), Arellano and Bond (1991), Arellano and Bover (1995) and (Blundell \& Bond, 1998) further refined this technique in dynamic panel data models. GMM technique results in unbiased consistent and efficient estimators through non-parametric approach and it further control for possible co-relations among independent variables (Schultz et al., 2010; Baltagi, 2008). Therefore, the problems of heteroscedasticity, simultaneity and dynamic endogeneity is accounted for.

This paper follows the model proposed by Wintoki et al. (2012), where CG has a dynamic relationship with firm performance and is a function of past performance and other firm characteristics. Thus, the dynamic model for $\mathrm{CG}$ is as follows;

$X i t=f(Y i, t-1, Y i, t-2 \ldots Y i, t-p, Z i, t, \mu i, \varepsilon i t) \ldots \ldots \ldots \ldots \ldots \ldots \ldots \ldots \ldots . .1$

Where, $X$ represents the board governance of firm ith in year tth; $Y$ represents the firm performance (social and financial); $Z$ represents the other control variables, $\mu$ denotes the unobserved time-invariant firm effects, $\varepsilon$ represents the random error term and $p$ is the number of lags of firm performance.

Based on the above equation 1, the estimations of the effect of $\mathrm{CG}$ on firm performance can be expressed as follows:

$Y i t=f(Y i, t-1, Y i, t-2 \ldots Y i, t-p, X i, t, Z i, t, \mu i, \varepsilon i t)$. 2

The key assumptions in this model are the use of lags as instrumental variables (IVs) (Wintoki et al., 2012). The benefit of using lag variables is that they control for potential simultaneity and reverse causality. This method is only designed for situations where there are "short T, large N" panels, which means a panel with few time periods and many individual firms (Roodman, 2009). As our data set comprise of 183 MFIs over the spam of nine years, this method is very appropriate, using the instrument based on the lag of the independent variables.

In this paper system GMM approach as developed by Blundell and Bond (1998), is used because it reduces the small sample biased unlike the differences GMM approach. Further as advocated by (Nguyen, Locke, \& Reddy, 2015a) it is suitable for dealing with consistent values of corporate governance in the model.

\section{Results and Discussion}

\begin{tabular}{|c|c|c|c|c|c|c|c|c|c|c|c|c|}
\hline & \multicolumn{2}{|c|}{ ROA } & \multicolumn{2}{|r|}{ OSS } & \multicolumn{2}{|c|}{ ROA } & \multicolumn{2}{|r|}{ OSS } & \multicolumn{2}{|c|}{ ROA } & \multicolumn{2}{|c|}{ OSS } \\
\hline & (1) $\mathbf{a}$ & (1) $\mathrm{b}$ & (2) $\mathrm{a}$ & (2) b & (3) $\mathbf{a}$ & (3) $b$ & (4) $a$ & (4) $b$ & (5) $a$ & (5) $b$ & (6) $a$ & (6) b \\
\hline LROA & & $\begin{array}{c}0.235^{*} \\
(2.01)\end{array}$ & & & & $\begin{array}{l}0.061^{* * *} \\
*(1.88)\end{array}$ & & & & $\begin{array}{l}0.063^{*} \\
* \\
(1.94)\end{array}$ & & \\
\hline LOSS & & & & $\begin{array}{l}0.183^{*} \\
* \\
(2.56)\end{array}$ & & & & $\begin{array}{l}0.177 * * \\
(2.54)\end{array}$ & & & & $\begin{array}{l}0.177^{* * *} \\
(2.54)\end{array}$ \\
\hline LWTB & & & & & & & & & & & & \\
\hline LAOL & & & & & & & & & & & & \\
\hline CGI & $\begin{array}{c}-.004 \\
(-0.36)\end{array}$ & $\begin{array}{l}-0.001 \\
(-0.12)\end{array}$ & $\begin{array}{l}-.017^{*} \\
(-1.76)\end{array}$ & $\begin{array}{l}-0.013 \\
(-1.40)\end{array}$ & $\begin{array}{l}-.004 \\
(-\end{array}$ & $\begin{array}{l}-0.003 \\
(-0.23)\end{array}$ & $\begin{array}{l}- \\
0.175\end{array}$ & $\begin{array}{l}-0.013 \\
(-1.40)\end{array}$ & \begin{tabular}{|l}
-0.004 \\
$(-0.37)$
\end{tabular} & $\begin{array}{l}-0.004 \\
(-0.30)\end{array}$ & - & $\begin{array}{l}-0.013 \\
(-1.42)\end{array}$ \\
\hline HDI & $\begin{array}{l}1.087^{*} \\
* * \\
(5.16)\end{array}$ & $\begin{array}{l}1.061 * * \\
*(5.24)\end{array}$ & $\begin{array}{l}.446^{* * *} \\
(2.31)\end{array}$ & $\begin{array}{l}0.405^{*} \\
* \\
(2.06)\end{array}$ & $\begin{array}{l}0.30) \\
.894 * * * \\
(3.46)\end{array}$ & $\begin{array}{l}0.945^{* * *} \\
*(3.62)\end{array}$ & $\begin{array}{l}* \\
0.701^{*} \\
* * \\
(3.58)\end{array}$ & $\begin{array}{l}0.653^{* * *} \\
*(3.31)\end{array}$ & \begin{tabular}{|l|}
$0.871^{*}$ \\
$* *$ \\
$(3.45)$
\end{tabular} & \begin{tabular}{|l}
$0.920 *$ \\
$* *$ \\
$(3.62)$
\end{tabular} & $\begin{array}{l}(-175) \\
0.698 * * \\
*(3.52)\end{array}$ & $\begin{array}{l}0.647 \text { *** } \\
(3.25)\end{array}$ \\
\hline GDPPC & $\begin{array}{l}.15^{* *} \\
*(-3.72)\end{array}$ & $\mid \begin{array}{l}.98 * * \\
* \\
(-3.60)\end{array}$ & $\begin{array}{c}-6.89 \\
(-0.88)\end{array}$ & \begin{tabular}{|l|}
-5.16 \\
$(-0.67)$
\end{tabular} & $\begin{array}{l}1.25^{* * * *} \\
(- \\
65)\end{array}$ & $\begin{array}{l}-1.35^{* *} \\
(-2.92)\end{array}$ & $\begin{array}{r}2.03 * * * \\
(-3.49)\end{array}$ & $\begin{array}{l}1.82 * * * \\
(-3.15)\end{array}$ & {$\left[\begin{array}{l}9.10^{*} \\
* \\
(-1.89)\end{array}\right.$} & \begin{tabular}{|c}
-9.91 *** \\
$(-2.09)$
\end{tabular} & $\begin{array}{l}1.97 * * \\
(-3.29)\end{array}$ & $\begin{array}{r}1.73 * * * \\
(-2.94)\end{array}$ \\
\hline
\end{tabular}




\begin{tabular}{|c|c|c|c|c|c|c|c|c|c|c|c|c|}
\hline GI & $\begin{array}{c}- \\
.006 * * \\
* \\
(-3.28)\end{array}$ & $\begin{array}{c}- \\
0.006^{*} \\
* \\
(-3.14)\end{array}$ & \begin{tabular}{c|}
-.002 \\
$(-1.11)$
\end{tabular} & $\begin{array}{l}-0.001 \\
(-0.81)\end{array}$ & $\begin{array}{l}- \\
.006^{*} \\
* * \\
(-\end{array}$ & $\begin{array}{l}- \\
0.007 * \\
* * \\
(-3.38)\end{array}$ & $\begin{array}{l}0.000 \\
(0.26)\end{array}$ & \begin{tabular}{|l}
0.000 \\
$(0.45)$
\end{tabular} & $\begin{array}{c}- \\
0.006^{*} \\
* * \\
(-3.16)\end{array}$ & $\begin{array}{c}- \\
0.006 * \\
* * \\
(-3.20)\end{array}$ & $\begin{array}{l}0.000 \\
(0.32)\end{array}$ & $\begin{array}{l}0.001 \\
(0.55)\end{array}$ \\
\hline $\begin{array}{l}\text { D } \\
\text { C }\end{array}$ & $\begin{array}{l}.001^{* *} \\
(2.16) \\
\end{array}$ & $\begin{array}{l}0.001^{*} \\
(1.73)\end{array}$ & $\begin{array}{c}-.000 \\
(-0.51) \\
\end{array}$ & $\begin{array}{l}-0.000 \\
(-0.50) \\
\end{array}$ & $\begin{array}{l}.000 \\
(1.42)\end{array}$ & $\begin{array}{l}0.000 \\
(1.39)\end{array}$ & $\begin{array}{l}0.001 * \\
(1.74)\end{array}$ & \begin{tabular}{|l|}
0.001 \\
$(1.62)$
\end{tabular} & $\begin{array}{l}0.001^{*} \\
(1.70)\end{array}$ & $\begin{array}{l}0.001 \\
(1.68)\end{array}$ & $\begin{array}{l}0.001^{*} \\
(1.83)\end{array}$ & $\begin{array}{l}0.001^{*} \\
(1.71)\end{array}$ \\
\hline $\begin{array}{l}\text { Inflatio } \\
\text { n }\end{array}$ & $\begin{array}{c}-002 * \\
* \\
(-2.63)\end{array}$ & $\begin{array}{l}0.002 \\
* \begin{array}{l}-0 \\
2.53)\end{array}(-\end{array}$ & $\begin{array}{c}-.000 \\
(-0.47)\end{array}$ & $\begin{array}{l}-0.000 \\
(-0.05)\end{array}$ & $\begin{array}{l}-.002^{* * * *} \\
(-2.37)\end{array}$ & $\begin{array}{l}-0.002 \\
(-2.37)\end{array}$ & $\begin{array}{l}-0.001 \\
(-1.01)\end{array}$ & \begin{tabular}{|l|}
-0.000 \\
$(-0.65)$
\end{tabular} & $\begin{array}{c}02 * * * \\
(-2.43)\end{array}$ & $\begin{array}{l}.002 * \\
* \\
(-2.43)\end{array}$ & $\begin{array}{l}-0.001 \\
(-0.99)\end{array}$ & $\begin{array}{l}-0.000 \\
(-0.63)\end{array}$ \\
\hline$\overline{\mathrm{DI}}$ & & & & & $\begin{array}{l}.020^{*} \\
(1.29)\end{array}$ & $\begin{array}{l}0.018 \\
(1.23)\end{array}$ & $\begin{array}{l}-0.010 \\
(-0.77)\end{array}$ & \begin{tabular}{|l|}
-0.011 \\
$(-0.90)$
\end{tabular} & $\begin{array}{l}0.038^{*} \\
* *\end{array}$ & $\begin{array}{l}0.037 * \\
*\end{array}$ & $\begin{array}{l}-0.007 \\
(-0.44)\end{array}$ & $\begin{array}{l}-0.007 \\
(-0.47)\end{array}$ \\
\hline CPI & & & & & $\begin{array}{l}.002^{*} \\
(1.45)\end{array}$ & \begin{tabular}{|l|}
0.002 \\
$(1.33)$
\end{tabular} & $\left|\begin{array}{l}0.009 * * \\
* \\
(-5.64)\end{array}\right|$ & $\mid \begin{array}{c}0.008 * * * \\
(-5.61)\end{array}$ & $\begin{array}{l}(2.56) \\
0.002 \\
(1.19)\end{array}$ & $\begin{array}{l}(2.57) \\
0.002 \\
(1.07)\end{array}$ & $\begin{array}{l}.009 * * \\
* \\
(-5.88)\end{array}$ & $\begin{array}{l}0.009 * * \\
* \\
(-5.84)\end{array}$ \\
\hline HI & & & & & & & & & $\begin{array}{l}.005^{*} \\
* \\
(-2.30)\end{array}$ & $\begin{array}{l}.005^{*} \\
* \\
(-2.41)\end{array}$ & $\begin{array}{l}-0.000 \\
(-0.38)\end{array}$ & $\begin{array}{l}-0.001 \\
(-0.60)\end{array}$ \\
\hline \begin{tabular}{|l} 
Age \\
\end{tabular} & $\begin{array}{c}.004 \\
(1.23)\end{array}$ & $\begin{array}{l}0.005 \\
(1.47)\end{array}$ & $\begin{array}{c}.000 \\
(0.19)\end{array}$ & $\begin{array}{l}0.002 \\
(0.54)\end{array}$ & $\begin{array}{l}.005^{*} \\
(1.70)\end{array}$ & $\begin{array}{l}0.006^{*} \\
(1.82)\end{array}$ & $\begin{array}{r}-0.000 \\
(-0.21)\end{array}$ & $\begin{array}{l}0.000 \\
(0.17)\end{array}$ & $\begin{array}{l}0.005^{*} \\
(1.53)\end{array}$ & $\begin{array}{l}0.005 \\
(1.66)\end{array}$ & $\begin{array}{l}-0.001 \\
(-0.24)\end{array}$ & $\begin{array}{l}0.000 \\
(0.14)\end{array}$ \\
\hline Age $^{2}$ & $\begin{array}{l}-.000 \\
(-1.02)\end{array}$ & $\begin{array}{l}-0.000 \\
(-1.44)\end{array}$ & $\begin{array}{c}-.000 \\
(-1.27)\end{array}$ & $\begin{array}{l}-0.000 \\
(-1.45)\end{array}$ & $\begin{array}{l}-.000^{*} \\
(-\end{array}$ & $\begin{array}{l}-0.000 \\
(-1.65)\end{array}$ & $\begin{array}{l}-0.000 \\
(-0.82)\end{array}$ & \begin{tabular}{|l|}
-0.000 \\
$(-1.05)$
\end{tabular} & $\begin{array}{l}-0.000 \\
(-1.25)\end{array}$ & $\begin{array}{l}-0.000 \\
(-1.43)\end{array}$ & $\begin{array}{l}-0.000 \\
(-0.79)\end{array}$ & $\begin{array}{l}-0.000 \\
(-1.01)\end{array}$ \\
\hline LAsse & $\begin{array}{l}-.034 * * \\
(-1.96)\end{array}$ & $\begin{array}{l}-0.026 \\
(-1.67)\end{array}$ & \begin{tabular}{c|}
.009 \\
$(1.39)$
\end{tabular} & $\begin{array}{l}0.006 \\
(0.99)\end{array}$ & $\begin{array}{l}1.48) \\
- \\
.035 * \\
* \\
(-\end{array}$ & $\begin{array}{l}- \\
0.034 * \\
* \\
(-1.98)\end{array}$ & $\begin{array}{l}0.013^{*} \\
(1.86)\end{array}$ & \begin{tabular}{|l|}
0.010 \\
$(1.42)$
\end{tabular} & $\begin{array}{c}- \\
0.035^{*} \\
* \\
(-2.02)\end{array}$ & $\begin{array}{c}- \\
0.034 * \\
* \\
(-1.97)\end{array}$ & $\begin{array}{l}0.013^{*} \\
(1.87)\end{array}$ & $\begin{array}{l}0.010 \\
(1.43)\end{array}$ \\
\hline $\begin{array}{l}\text { Regula } \\
\text { ted }\end{array}$ & $\begin{array}{l}0.083 * \\
* * \\
(283)\end{array}$ & $\begin{array}{l}0.067^{*} \\
(2.42)\end{array}$ & $\begin{array}{l}0.000 \\
(0.01)\end{array}$ & $\begin{array}{l}0.007 \\
(0.16)\end{array}$ & $\begin{array}{l}.080^{*} \\
* \\
(251)\end{array}$ & $\begin{array}{l}0.075^{*} \\
* \\
(234)\end{array}$ & $\begin{array}{l}-0.019 \\
(-0.41)\end{array}$ & $\begin{array}{l}-0.010 \\
(-0.21)\end{array}$ & $\begin{array}{l}0.075 * \\
* * \\
(241)\end{array}$ & $\begin{array}{l}0.070 * \\
* \\
(222)\end{array}$ & $\begin{array}{l}-0.020 \\
(-0.42)\end{array}$ & $\begin{array}{l}-0.011 \\
(-0.24)\end{array}$ \\
\hline \begin{tabular}{|l|} 
Rura \\
bank
\end{tabular} & $\begin{array}{l}-.157 \\
(-1.73)\end{array}$ & $\begin{array}{l}-0.112 \\
(-1.34)\end{array}$ & $\begin{array}{l}.209 * \\
* \\
* \\
\end{array}$ & $\begin{array}{l}0.186^{*} \\
* * \\
(437)\end{array}$ & $\begin{array}{l}1.160^{*} \\
(- \\
1.78) \\
\end{array}$ & \begin{tabular}{|l}
1 \\
-0.148 \\
$(-1.70)$
\end{tabular} & $\begin{array}{l}0.232 * \\
* * \\
(551\end{array}$ & \begin{tabular}{|l}
$0.207 *$ \\
$* *$ \\
$(481)$ \\
\end{tabular} & $\begin{array}{l}- \\
0.151^{*} \\
(-170)\end{array}$ & $\begin{array}{l}(2.22) \\
-0.139 \\
(-1.61)\end{array}$ & $\begin{array}{l}0.233^{*} \\
* * \\
(561) \\
\end{array}$ & $\begin{array}{l}0.210^{*} \\
* * \\
(489)\end{array}$ \\
\hline NBFI & $\begin{array}{c}- \\
.170 * * \\
* \\
(-2.49)\end{array}$ & $\begin{array}{c}0.139 * \\
* \\
(-2.15)\end{array}$ & \begin{tabular}{l}
\multicolumn{1}{c}{3} \\
$.065^{*}$ \\
$*$ \\
$(2.18)$
\end{tabular} & $\begin{array}{l}0.056^{*} \\
(1.88)\end{array}$ & $\begin{array}{l}- \\
.171 * \\
* * \\
(-\end{array}$ & $\begin{array}{l}- \\
0.164 * \\
* \\
(-2.55)\end{array}$ & $\begin{array}{l}0.084 * \\
* * \\
(2.79 \\
)\end{array}$ & $\begin{array}{l}0.074 * \\
* \\
(2.44)\end{array}$ & $\begin{array}{c}0.165 * \\
* * \\
(-2.52)\end{array}$ & $\begin{array}{c}- \\
0.158^{*} \\
* \\
(-2.48)\end{array}$ & $\begin{array}{l}0.085 * \\
* * \\
(2.83)\end{array}$ & $\begin{array}{l}0.075^{*} \\
*(2.49)\end{array}$ \\
\hline NGO & $\begin{array}{l}-.163^{* *} \\
(-2.20)\end{array}$ & $\begin{array}{c}- \\
0.138 * \\
* \\
(-1.99)\end{array}$ & $\begin{array}{l}4 \\
.166 * \\
* \\
*\end{array}$ & $\begin{array}{l}0.146^{*} \\
* * \\
(2.80)\end{array}$ & $\begin{array}{l}--\infty \\
.163 * \\
* \\
(-\end{array}$ & $\begin{array}{l}- \\
0.159 * \\
* \\
(-2.21)\end{array}$ & $\begin{array}{l}0.165 * \\
* * \\
(3.10 \\
)\end{array}$ & \begin{tabular}{|l}
$0.145^{*}$ \\
$* *$ \\
$(2.84)$
\end{tabular} & $\begin{array}{c}- \\
0.155^{*} \\
* \\
(-2.13)\end{array}$ & $\begin{array}{c}- \\
0.151 * \\
* \\
(-2.11)\end{array}$ & \begin{tabular}{|l}
$0.166^{*}$ \\
$* *$ \\
$(3.18)$
\end{tabular} & $\begin{array}{l}0.147 * \\
* * \\
(2.90)\end{array}$ \\
\hline \begin{tabular}{|l|} 
Credit \\
Union
\end{tabular} & $\begin{array}{c}- \\
.224 * * \\
* \\
(-2.73)\end{array}$ & $\begin{array}{c}- \\
0.190 * \\
* \\
(-2.55)\end{array}$ & $\begin{array}{l}0.198 \\
5.1 .26)\end{array}$ & $\begin{array}{l}0.161 \\
(1.02)\end{array}$ & $\begin{array}{l}- \\
-198 * \\
.198 \\
* * \\
(-\end{array}$ & \begin{tabular}{|l|}
- \\
$0.193 *$ \\
$*$ \\
$(-2.42)$
\end{tabular} & $\begin{array}{l}0.146 \\
(0.91)\end{array}$ & \begin{tabular}{|l|}
0.113 \\
$(0.70)$
\end{tabular} & $\begin{array}{c}- \\
0.1707 \\
* * \\
(-2.20)\end{array}$ & $\begin{array}{c}- \\
0.164 * \\
* \\
(-2.17)\end{array}$ & $\begin{array}{l}0.151 \\
(0.94)\end{array}$ & $\begin{array}{l}0.120 \\
(0.75)\end{array}$ \\
\hline $\begin{array}{l}\text { Group } \\
\text { Lendin } \\
\mathbf{g}\end{array}$ & $\begin{array}{l}.035 \\
(1.19)\end{array}$ & $\begin{array}{l}0.057^{*} \\
(1.86)\end{array}$ & $\begin{array}{l}- \\
.128 * \\
* \\
*\end{array}$ & $\begin{array}{c}- \\
0.120^{*} \\
* *\end{array}$ & $\begin{array}{l}.031 \\
(1.06)\end{array}$ & $\begin{array}{l}0.038 \\
(1.31)\end{array}$ & $\begin{array}{l}- \\
0.108 * \\
* *\end{array}$ & $\begin{array}{l}0.104 * \\
* *\end{array}$ & $\begin{array}{l}0.038 \\
(1.25)\end{array}$ & $\begin{array}{l}0.046 \\
(1.51)\end{array}$ & $\begin{array}{c}0.107^{*} \\
* *\end{array}$ & $\begin{array}{c}- \\
0.102 * \\
* *\end{array}$ \\
\hline $\begin{array}{l}\text { Individ } \\
\text { ual and } \\
\text { Group } \\
\text { lending }\end{array}$ & $\begin{array}{c}.030 \\
(1.11)\end{array}$ & $\begin{array}{l}0.033 \\
(1.24)\end{array}$ & \begin{tabular}{|c|}
\multicolumn{2}{c|}{-} \\
$.088^{*}$ \\
$* \quad(-$ \\
$2.39)$
\end{tabular} & $\begin{array}{c}0.078^{*} \\
* \\
(-2.13)\end{array}$ & \begin{tabular}{|l|}
.0373 \\
$(1.46)$
\end{tabular} & \begin{tabular}{|l|}
0.038 \\
$(1.52)$
\end{tabular} & $\begin{array}{l}- \\
0.097 * \\
* \\
(-2.57)\end{array}$ & $\begin{array}{l}- \\
0.088 * \\
* \\
(-2.32)\end{array}$ & $\begin{array}{l}0.040^{*} \\
(1.54)\end{array}$ & $\begin{array}{l}0.041 \\
(1.60)\end{array}$ & $\begin{array}{c}0.097 * \\
* * \\
(-2.55)\end{array}$ & $\begin{array}{l}0.087^{*} \\
* \\
(-2.30)\end{array}$ \\
\hline \begin{tabular}{|l|} 
Consta \\
nt
\end{tabular} & $\begin{array}{c}.350 \\
(1.12)\end{array}$ & $\begin{array}{l}0.161 \\
(0.55)\end{array}$ & $\begin{array}{l}.930^{*} \\
* \\
*\end{array}$ & $\begin{array}{l}0.717 * \\
* * \\
(3.19)\end{array}$ & $\begin{array}{l}.413 \\
(1.33)\end{array}$ & $\begin{array}{l}0.365 \\
(1.20)\end{array}$ & $\begin{array}{l}0.831 * \\
* * \\
(3.79\end{array}$ & $\begin{array}{l}0.629 * \\
* * \\
(2.80)\end{array}$ & $\begin{array}{l}0.654^{*} \\
(1.82)\end{array}$ & $\begin{array}{l}0.613^{*} \\
(1.73)\end{array}$ & \begin{tabular}{|l|}
$0.873^{*}$ \\
$* *$ \\
$(3.21)$
\end{tabular} & $\begin{array}{l}0.690^{*} \\
*(2.55)\end{array}$ \\
\hline
\end{tabular}




\begin{tabular}{|r|c|c|c|c|c|c|c|c|c|c|c|c|}
\hline $\begin{array}{r}\text { No. of } \\
\text { Obs }\end{array}$ & 1593 & 1592 & 1531 & 1510 & 1593 & 1591 & 1531 & 1510 & 1593 & 1591 & 1531 & 1510 \\
\hline F Value & 2.84 & 2.86 & 4.57 & 4.92 & 2.55 & 2.46 & 5.26 & 5.72 & 2.42 & 2.34 & 5.58 & 6.15 \\
\hline Prob & 0.0001 & 0.000 & 0.0000 & 0.000 & 0.000 & 0.000 & 0.000 & 0.000 & 0.000 & 0.000 & 0.000 & 0.000 \\
\hline
\end{tabular}

$*, * * \& * * *$ denote significance level at $10,5 \& 1 \%$ respectively

Table 4.1 presents the results of impact of corporate governance on financial performance using GMM analysis. Considering the dynamic nature of this relationship the analysis indicates the persistence of financial performance where the past financial performance significance determine the variation in the current performance. Therefore, to control for this dynamic endogeneity two year lag financial performance variable included as explanatory variables in all (1b-6b) equations. Overall the results confirm insignificance impact of corporate governance on financial performance even controlling for endogeneity. Through, in particular corporate governance has significant negative impact on financial performance when proxies by OSS. In economic variables the human development and domestic credit contributes to financial performance whereas per capita GDP, globalization index and inflation reduces it. Further, evidence found that size of MFI has inclusive impact on financial performance. Further, MFIs which are regulated having bank status financially perform better. Further, some evidence has been found that the institutional variables of having more democratic values and less perception about corruption prevailing in the society leads to better financial performance (eq 3-4). Furthermore, little evidence has found that cultural values undermine the financial performance (eq 5-6).

Table 4.2: CGI and Social performance

\begin{tabular}{|c|c|c|c|c|c|c|c|c|c|c|c|c|}
\hline & \multicolumn{2}{|l|}{ WTB } & \multicolumn{2}{|r|}{ AOL } & \multicolumn{2}{|c|}{ WT } & \multicolumn{2}{|r|}{ AOL } & \multicolumn{2}{|r|}{ WTB } & \multicolumn{2}{|r|}{ AOL } \\
\hline & (1) $\mathrm{a}$ & (1) $\mathrm{b}$ & (2) $\mathrm{a}$ & (2) b & (3) $\mathrm{a}$ & (3) $\mathrm{b}$ & (4) $\mathrm{a}$ & (4) $b$ & (5) $\mathrm{a}$ & (5) $\mathrm{b}$ & (6) $\mathrm{a}$ & (6) b \\
\hline LROA & & & & & & & & & & & & \\
\hline LOSS & & & & & & & & & & & & \\
\hline LWTB & & $\begin{array}{l}0.401 * \\
* \\
*\end{array}$ & & & & $\begin{array}{l}0.399 * * \\
* \\
(15.8\end{array}$ & & & & $\begin{array}{l}0.386^{*} \\
* \\
*\end{array}$ & & \\
\hline LAOL & & & & $\begin{array}{l}0.426 * \\
* \\
*\end{array}$ & & & & $\begin{array}{l}0.418^{* *} \\
* \\
(6.33)\end{array}$ & & & & $\begin{array}{l}0.418^{*} \\
* \\
*\end{array}$ \\
\hline $\begin{array}{l}\text { C } \\
\text { GI }\end{array}$ & $\begin{array}{l}.014 * * \\
* \\
057)\end{array}$ & $\begin{array}{l}0.011 * \\
* \\
(244)\end{array}$ & $\begin{array}{l}- \\
.064 * * \\
* \quad(- \\
\end{array}$ & 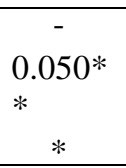 & $\begin{array}{l}0.012 \text { ** } \\
(2.25)\end{array}$ & $\begin{array}{l}0.010^{* * *} \\
(2.13)\end{array}$ & $\begin{array}{l}- \\
0.062 * * \\
* \\
(-512)\end{array}$ & $\begin{array}{l}0.050 * \\
* * \quad(- \\
450)\end{array}$ & $\begin{array}{l}0.013 * \\
* \\
*\end{array}$ & $\begin{array}{l}0.011^{*} \\
* \\
(238)\end{array}$ & $\begin{array}{l}0.063 * \\
* \\
\quad *\end{array}$ & $\begin{array}{l}0.050 * \\
* \\
*\end{array}$ \\
\hline $\begin{array}{l}\text { HD } \\
\text { I }\end{array}$ & $\begin{array}{l}1.542 * \\
* \\
*\end{array}$ & $\begin{array}{l}1 . \\
1.170 * \\
* \\
* \\
\end{array}$ & $\begin{array}{l}.527 * \\
* \\
0 \cap 8\end{array}$ & $\begin{array}{l}0.08 \\
8 \\
63\end{array}$ & $\begin{array}{l} \\
1.454 * * \\
*\end{array}$ & $\begin{array}{l}- \\
1.12 * * \\
* \quad(- \\
100 \Omega \\
\end{array}$ & $\begin{array}{l}0.880 * * \\
* \\
(3.3 \\
7\end{array}$ & $\begin{array}{l}0.1465^{*} \\
(1.82)\end{array}$ & \begin{tabular}{c}
\multicolumn{2}{c}{-} \\
$1.393 *$ \\
$*$ \\
$*$ \\
\end{tabular} & $\begin{array}{l}1 . \\
1.085^{*} \\
* \\
* \\
\end{array}$ & $\begin{array}{l}0.865^{*} \\
* \\
*\end{array}$ & $\begin{array}{l}0.462 \\
* \\
1777\end{array}$ \\
\hline $\begin{array}{l}\text { GDPP } \\
\text { C }\end{array}$ & $\begin{array}{c}3.32 * * \\
*\end{array}$ & $\begin{array}{c}2.64 * * \\
*\end{array}$ & $\begin{array}{l}- \\
3.68 * * \\
* \quad(-\end{array}$ & $\begin{array}{l}-1.20 \\
(-1.41)\end{array}$ & $\begin{array}{l}3.08^{* * * *} \\
(5.16)\end{array}$ & $\begin{array}{l}2.57 * * * * \\
(5.26)\end{array}$ & $\begin{array}{l}- \\
5.67 * * \\
* \quad(-\end{array}$ & $\begin{array}{l}-3.20 * * * \\
(-2.72)\end{array}$ & $\begin{array}{l}2.47 * * \\
*\end{array}$ & $\begin{array}{c}2.13 * * \\
*\end{array}$ & $\begin{array}{l} \\
\\
5.44 * * \\
* \quad(-\end{array}$ & $3.15^{*}$ \\
\hline $\begin{array}{l}\text { G } \\
\text { I }\end{array}$ & $\begin{array}{l}-.000 \\
(-0.45)\end{array}$ & $\begin{array}{l}- \\
0.000\end{array}$ & $\begin{array}{l}- \\
.009 * * \\
* \quad(-\end{array}$ & $\begin{array}{l}- \\
0.007 * \\
* \quad(-\end{array}$ & $\begin{array}{l}0.002^{*} \\
(-1.85)\end{array}$ & $\begin{array}{l}- \\
0.002 * \\
* \quad(- \\
205)\end{array}$ & $\begin{array}{l}-0.004 \\
(-1.39)\end{array}$ & $\begin{array}{l}-0.003 \\
(-1.02)\end{array}$ & $\begin{array}{c}- \\
0.003 * \\
* \\
*\end{array}$ & $\begin{array}{l} \\
\\
0.003 * \\
* \\
* \\
*\end{array}$ & $\begin{array}{l}- \\
0.004 \\
c_{-}\end{array}$ & $\begin{array}{l}- \\
0.003 \\
c_{-}\end{array}$ \\
\hline $\begin{array}{l}\text { D } \\
\text { C }\end{array}$ & $\begin{array}{l}.005^{* *} \\
*\end{array}$ & $\begin{array}{l}0.003 * \\
* \\
*\end{array}$ & ${ }^{-} .001 *$ & $\begin{array}{l}- \\
0.001 * \\
* \quad(-\end{array}$ & $\begin{array}{l}0.004 * * \\
* \\
(10.4\end{array}$ & $\begin{array}{l}0.003 * * \\
* \\
(8.6\end{array}$ & $\begin{array}{l}0.001 \\
(0.99)\end{array}$ & $\begin{array}{l}0.000 \\
(0.62)\end{array}$ & $\begin{array}{l}0.003 * \\
* \\
*\end{array}$ & $\begin{array}{l}0.002 * \\
* \\
*\end{array}$ & $\begin{array}{l}0.00 \\
1\end{array}$ & $\begin{array}{l}0.00 \\
0\end{array}$ \\
\hline $\begin{array}{l}\text { Inflatio } \\
\text { n }\end{array}$ & $\begin{array}{c}-.000 \\
(-0.87)\end{array}$ & $\begin{array}{l}-0.000 \\
(-0.73)\end{array}$ & $\begin{array}{l}.006 * * \\
* \\
(-3.40)\end{array}$ & $\begin{array}{l}0 . \\
0.006^{*} \\
* \\
\quad * \\
(- \\
5.61)\end{array}$ & $\begin{array}{l}-0.000 \\
(-1.24)\end{array}$ & $\begin{array}{l}-0.000 \\
(-0.90)\end{array}$ & $\begin{array}{l}0.007 * * \\
* \\
(-3.05)\end{array}$ & $\begin{array}{l}0.007 * * \\
* \\
(-5.91)\end{array}$ & $\begin{array}{c}0.000^{*} \\
(-1.63)\end{array}$ & $\begin{array}{l}-0.000 \\
(-1.10)\end{array}$ & $\begin{array}{l}0.007 * \\
* \\
* \\
(- \\
3.08)\end{array}$ & $\begin{array}{l}0.007 * \\
*{ }^{*} \\
\quad * \\
(- \\
5.93)\end{array}$ \\
\hline
\end{tabular}




\begin{tabular}{|c|c|c|c|c|c|c|c|c|c|c|c|c|}
\hline $\begin{array}{l}\text { D } \\
\text { I }\end{array}$ & & & & & $\begin{array}{l}0.023^{* *} \\
* \\
(-4.29)\end{array}$ & $\begin{array}{l}- \\
0.018 * * * \\
* \\
(-402)\end{array}$ & $\begin{array}{l}-0.005 \\
(-0.33)\end{array}$ & $\begin{array}{l}-0.016 \\
(-1.02)\end{array}$ & $\begin{array}{l}0.052 * \\
* \\
\quad *\end{array}$ & $\begin{array}{l} \\
0.040 * \\
* \\
*\end{array}$ & $\begin{array}{l}0.00 \\
6 \\
(\cap 02\end{array}$ & $\begin{array}{l}- \\
0.014 \\
\text { (- }\end{array}$ \\
\hline $\begin{array}{l}\text { CP } \\
\text { I }\end{array}$ & & & & & $\begin{array}{l}0.004 * * \\
* \\
(4.2 \\
0\end{array}$ & $\begin{array}{l}0.004 * * \\
* \\
(5.0 \\
0 .\end{array}$ & $\begin{array}{l}0.017 * * \\
* \\
1 \quad 02\end{array}$ & $\begin{array}{l}- \\
0.013 * \\
* * \quad(- \\
5.17)\end{array}$ & $\begin{array}{l}0.005^{*} \\
* \\
*\end{array}$ & $\begin{array}{l}0.005^{*} \\
* \\
*\end{array}$ & $\begin{array}{l}0.017 * \\
* \\
\quad * \\
\end{array}$ & $\begin{array}{l}- \\
0.013 * \\
* \quad(- \\
\end{array}$ \\
\hline $\begin{array}{l}\mathbf{H} \\
\mathbf{I}\end{array}$ & & & & & & & & & $\begin{array}{l}0.009^{*} \\
* \\
* \\
\end{array}$ & $\begin{array}{l}0.006^{*} \\
* \\
*\end{array}$ & - & $\begin{array}{l}0.000^{*} \\
* \quad(-\end{array}$ \\
\hline $\begin{array}{l}\text { Ag } \\
\text { e }\end{array}$ & $\begin{array}{r}- \\
.006^{* *} \\
* \quad(- \\
\end{array}$ & $\begin{array}{l}- \\
0.002 \\
{ }_{-}\end{array}$ & $\begin{array}{l}.016 * * \\
* \\
(276)\end{array}$ & $\begin{array}{l}0.018 * \\
* \\
*\end{array}$ & $\begin{array}{l}- \\
0.006 * * \\
* \\
(-264)\end{array}$ & $\begin{array}{l}-0.003 \\
(-1.23)\end{array}$ & $\begin{array}{l}0.013 * * \\
* \\
(2.4 \\
9) \\
9\end{array}$ & $\begin{array}{l}0.015 * * \\
(2.59)\end{array}$ & \begin{tabular}{|r|}
\multicolumn{2}{|c}{-} \\
$0.005^{*}$ \\
$* \quad(-$
\end{tabular} & $\begin{array}{l}- \\
0.00 \\
2 \quad 1\end{array}$ & $\begin{array}{l}0.013 * \\
* \\
\text { (2 39) }\end{array}$ & $\begin{array}{l}0.015 * \\
* \\
(256)\end{array}$ \\
\hline $\operatorname{Age}^{2}$ & $\begin{array}{l}.000 * * \\
* \\
(510)\end{array}$ & $\begin{array}{l}0.000 * \\
* \\
*\end{array}$ & $\begin{array}{l}- \\
.000^{* *} \\
* \quad(-\end{array}$ & $\begin{array}{l}- \\
0.000^{*} \\
* \\
*\end{array}$ & $\begin{array}{l}0.000 * * \\
* \\
(5.4\end{array}$ & $\begin{array}{l}0.000 * * \\
* \\
(3.4\end{array}$ & $\begin{array}{l}0.000 * * \\
*\end{array}$ & $\begin{array}{l}- \\
0.000 * \\
* * \quad(-\end{array}$ & $\begin{array}{l}0.000^{*} \\
* \\
*\end{array}$ & $\begin{array}{l}0.000 * \\
* \\
*\end{array}$ & $\begin{array}{l}- \\
0.000 * \\
*\end{array}$ & $\begin{array}{l}- \\
0.000^{*} \\
*\end{array}$ \\
\hline LAsset & $\begin{array}{c}-.000 \\
(-0.13) \\
\end{array}$ & $\begin{array}{l}-0.002 \\
(-0.71) \\
\end{array}$ & $\begin{array}{l}.010 \\
(1.01) \\
\end{array}$ & $\begin{array}{l}0.016^{*} \\
(1.89)\end{array}$ & \begin{tabular}{|l|}
-0.002 \\
$(-0.63)$ \\
\end{tabular} & $\begin{array}{l}70.004 \\
(-1.33) \\
\end{array}$ & $\begin{array}{l}200 \\
0.016^{*} \\
(1.57)\end{array}$ & $\begin{array}{l}2.021 * * \\
(2.39)\end{array}$ & $\begin{array}{r}-0.003 \\
(-0.89) \\
\end{array}$ & $\begin{array}{l}-0.004 \\
(-1.57) \\
\end{array}$ & $\begin{array}{l}0.016^{*} \\
(1.58)\end{array}$ & $0.021^{*}$ \\
\hline $\begin{array}{r}\text { Regula } \\
\text { te d }\end{array}$ & \begin{tabular}{l}
\multicolumn{2}{c}{-} \\
$.053 * *$ \\
$* \quad(-$ \\
\end{tabular} & \begin{tabular}{l}
\multicolumn{2}{c}{-} \\
$0.040^{*}$ \\
$* \quad(-$ \\
\end{tabular} & $\begin{array}{l}.115^{* *} \\
*\end{array}$ & $\begin{array}{l}0.04 \\
2\end{array}$ & $\begin{array}{l}0.031^{*} \\
(-1.75) \\
\end{array}$ & $\begin{array}{l}-0.020 \\
(-1.36)\end{array}$ & $\begin{array}{l}0.074 * * \\
* \\
(2.5\end{array}$ & $\begin{array}{l}0.016^{* *} \\
* \\
* \\
\end{array}$ & $\begin{array}{l}- \\
0.024\end{array}$ & $\begin{array}{l}- \\
0.016\end{array}$ & $\begin{array}{l}0.070^{*} \\
* \\
*\end{array}$ & $\begin{array}{l}0.01 \\
6\end{array}$ \\
\hline $\begin{array}{l}\text { Rur } \\
\text { al } \\
\text { han }\end{array}$ & $\begin{array}{l}.157 * * \\
* \\
(435) \\
\end{array}$ & $\begin{array}{l}0.108^{*} \\
* \\
*\end{array}$ & $\begin{array}{l}- \\
.584 * * \\
* \quad(- \\
\end{array}$ & $\begin{array}{l}0.421 * \\
* \\
\quad * \\
\end{array}$ & $\begin{array}{l}0.139 * * \\
* \\
(3.8 \\
1) \\
\end{array}$ & $\begin{array}{l}0.091 * * \\
* \\
(3.2 \\
0) \\
\end{array}$ & $\begin{array}{l}0.541 * * \\
* \\
(-618)\end{array}$ & $\begin{array}{l}0.393 * \\
* * \quad(- \\
542)\end{array}$ & $\begin{array}{l}0.119^{*} \\
* \\
*\end{array}$ & $\begin{array}{l}0.078 * \\
* \\
*\end{array}$ & $\begin{array}{l}0.536^{*} \\
* \\
\quad * \\
\end{array}$ & $\begin{array}{l}0.392 * \\
* \\
\quad * \\
\end{array}$ \\
\hline NBFI & $\begin{array}{l}.220 * * \\
* \\
61180\end{array}$ & $\begin{array}{l}0.175^{*} \\
* \\
*\end{array}$ & $\begin{array}{r}- \\
.482 * * \\
* \quad(- \\
\end{array}$ & 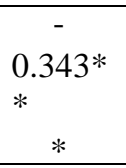 & $\begin{array}{l}0.204 * * \\
* \\
(10.5 \\
8\end{array}$ & $\begin{array}{l}0.160 * * \\
* \\
(8.8 \\
8) \\
\end{array}$ & $\begin{array}{l}- \\
0.447 * * \\
* \\
(-531)\end{array}$ & $\begin{array}{l}- \\
0.321 * \\
* * \quad(- \\
481)\end{array}$ & $\begin{array}{l}0.192 * \\
* \\
*\end{array}$ & $\begin{array}{l}0.153 * \\
* \\
*\end{array}$ & 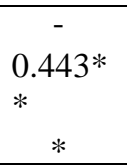 & $\begin{array}{l} \\
\\
0.320 * \\
* \\
* \\
\end{array}$ \\
\hline NGO & $\begin{array}{c}4 \\
.178^{* *} \\
*\end{array}$ & $\begin{array}{l}0.128^{*} \\
* \\
*\end{array}$ & $\begin{array}{r}- \\
.554 * * \\
* \quad(- \\
\end{array}$ & 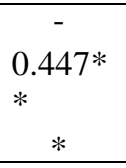 & $\begin{array}{l}0.176^{* *} \\
* \\
(7.5 \\
3 \\
\end{array}$ & $\begin{array}{l}0.126^{* *} \\
* \\
(6.1 \\
0 \\
\end{array}$ & $\begin{array}{l}- \\
0.552 * * \\
* \\
(-694)\end{array}$ & $\begin{array}{l}- \\
0.448 * \\
* * \quad(- \\
675)\end{array}$ & $\begin{array}{l}0.159^{*} \\
* \\
*\end{array}$ & $\begin{array}{l}0.115^{*} \\
* \\
*\end{array}$ & 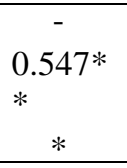 & $\begin{array}{l} \\
\\
0.447 * \\
* \\
* \\
\end{array}$ \\
\hline $\begin{array}{l}\text { Credit } \\
\text { Union }\end{array}$ & $\begin{array}{c}5 \\
.226 * * \\
*\end{array}$ & $\begin{array}{l}0.164 * \\
* \\
*\end{array}$ & $\begin{array}{l}- \\
.452 * * \\
* \quad(- \\
\end{array}$ & $\begin{array}{l}- \\
0.299 * \\
* \\
* \\
\end{array}$ & $\begin{array}{l}0.229 * * \\
* \\
(6.8 \\
6\end{array}$ & $\begin{array}{l}0.172 * * \\
* \\
(5.8 \\
6 \\
\end{array}$ & $\begin{array}{l}- \\
0.534 * * \\
* \\
(-582)\end{array}$ & $\begin{array}{l}0.375^{*} \\
* * \quad(- \\
486)\end{array}$ & $\begin{array}{l}0.180 * \\
* \\
*\end{array}$ & $\begin{array}{l}0.138 * \\
* \\
*\end{array}$ & $\begin{array}{l}0.516 * \\
* \\
\quad *\end{array}$ & $\begin{array}{l}0.372 * \\
* \\
*\end{array}$ \\
\hline $\begin{array}{l}\text { Group } \\
\text { Lendin } \\
\text { g }\end{array}$ & $\begin{array}{l}.142 * * \\
* \\
7727)\end{array}$ & $\begin{array}{l}0.079 * \\
* \\
*\end{array}$ & $\begin{array}{r}- \\
.286^{* *} \\
* \quad(- \\
\end{array}$ & $\begin{array}{l} \\
\\
0.195 * \\
* \\
* \\
\end{array}$ & $\begin{array}{l}0.133 * * \\
* \\
(6.8 \\
1) \\
\end{array}$ & $\begin{array}{l}0.069 * * \\
* \\
(3.8 \\
4) \\
\end{array}$ & $\begin{array}{l}0.257 * * \\
* \\
(-697)\end{array}$ & $\begin{array}{l}- \\
0.175 * \\
* * \quad(- \\
454)\end{array}$ & $\begin{array}{l}0.119 * \\
* \\
*\end{array}$ & $\begin{array}{l}0.061 * \\
* \\
*\end{array}$ & 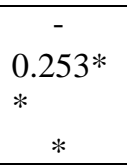 & 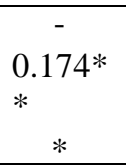 \\
\hline $\begin{array}{l}\text { Indivi } \\
\text { du } \\
\text { al } \\
\text { and }\end{array}$ & $\begin{array}{l}.121 * * \\
* \\
(792)\end{array}$ & $\begin{array}{l}0.084 * \\
* \\
* \\
\end{array}$ & $\begin{array}{l}- \\
.066^{*} \\
1-\end{array}$ & $\begin{array}{l}- \\
0.065 \\
{ }_{-}\end{array}$ & $\begin{array}{l}0.118 * * \\
* \\
(7.7 \\
5)\end{array}$ & $\begin{array}{l}0.083^{* *} \\
* \\
5.8 \\
5)\end{array}$ & $\begin{array}{ll}0.080 * \\
* \quad(- \\
193)\end{array}$ & $\begin{array}{l}-0.079^{* *} \\
(-2.07)\end{array}$ & $\begin{array}{l}0.112 * \\
* \\
* \\
\end{array}$ & $\begin{array}{l}0.080^{*} \\
* \\
*\end{array}$ & $\begin{array}{l}- \\
0.079 \\
*\end{array}$ & $\begin{array}{l}- \\
0.078^{*} \\
* \quad(-\end{array}$ \\
\hline $\begin{array}{l}\text { Consta } \\
\text { nt }\end{array}$ & $\begin{array}{l}1.181^{*} \\
* \\
*\end{array}$ & $\begin{array}{l}0.810^{*} \\
* \\
*\end{array}$ & $\begin{array}{l}1.194 * \\
* \\
*\end{array}$ & $\begin{array}{l}0.860 * \\
* \\
*\end{array}$ & $\begin{array}{l}1.181 * * \\
* \\
(12.0\end{array}$ & $\begin{array}{l}0.825 * * \\
* \\
(9.0\end{array}$ & $\begin{array}{l}1.038^{* * *} \\
* \\
(4.2\end{array}$ & $\begin{array}{l}0.717 \text { ** } \\
(2.99)\end{array}$ & \begin{tabular}{|l}
$0.818^{*}$ \\
$*$ \\
$*$ \\
\end{tabular} & $\begin{array}{l}0.574 * \\
* \\
*\end{array}$ & $\begin{array}{l}l^{*} .197^{*} \\
* \\
*\end{array}$ & $\begin{array}{l}0.752 * \\
* \\
*\end{array}$ \\
\hline $\begin{array}{l}\text { No. of } \\
\text { Obs. }\end{array}$ & 1575 & 1565 & 1594 & 1591 & 1575 & 1565 & 1594 & 1591 & 1575 & 1565 & 1594 & 1591 \\
\hline $\begin{array}{l}\text { Value } \\
\text { of }\end{array}$ & 153.15 & 233.16 & 23.69 & 27.00 & 132.70 & 207.37 & 21.20 & 24.29 & 157.94 & 227.07 & 21.61 & 25.52 \\
\hline Prob > & 0.0000 & 0.000 & 0.0000 & 0.000 & 0.000 & 0.000 & 0.000 & 0.000 & 0.000 & 0.000 & 0.000 & 0.000 \\
\hline
\end{tabular}

$*, * * \& * * *$ denote significance level at $10,5 \& 1 \%$ respectively

Evidence related to the impact of corporate governance on social performance is present in table 4.2. Like the financial performance analysis, the significant co-efficient of lagged social performance proxy depict the persistence nature of social performance variable where the past 
social performance effects the present social performance. Therefore, two year lagged social performance variable is included as explanatory variables in all equations (1b-6b). Overall the evidence suggests that unlike corporate governance-financial performance relationship, the good corporate governance significantly increases the social performance as proxies by women borrowers and average loan size of MFIs controlling for economic, institutional and cultural variables. Considering economic variables, per capita income enhances the social performance whereas surprisingly human development undermines the social performance. The result suggests that MFIs better serve their social agenda in areas having low level of human development.

Similarly, the results suggest that globalization reduces the social performance through less focus on female clients. Moreover, increase inflation also causes MFIs to focus on poor clients. The evidence further suggests that increase in domestic credit do significantly increase the social performance. The evidence related to institutional variable proxies by democracy index and CPI provides mixed evidence. This suggests that more democratic values lead to decrease in social performance through targeting less female clients. Whereas, lower level of corruption perception in the society contribute towards social performance. Interesting the un-linearity of experience of an MFIs with its social performance is empirically established which suggests that mature MFIs tend to more focus on social performance unlike the young MFIs. The evidence related to culture variable suggests that good culture values promote the social performance. Considering the categorical variables MFIs having bank status have individual lending methodology and those who are regulated have lower social performance.

\section{Conclusion}

This paper investigates the relationship of corporate governance and performance of microfinance institutions controlling for institutional, cultural and economic factors by utilizing a panel data set comprised of 183 MFIs in Asia from 2010 to 2018. It employs in addition to OLS, the GMM method to take care of the issue of endogeneity. A comprehensive CGI comprised of seven dimensions has been constructed. Comparing the CGI and performance relationship our results suggests that good corporate governance practices significantly increase the social performance of MFIs. Whereas some evidence has found that it undermines the financial performance. The result is robust even after controlling for economic, institutional and cultural factors. In the backdrop of agency theory and resource dependence theory we conclude that presence of international directors, board diversity and their qualification, female representation in the board and non-profit ownership structure results in more lending to female clients and targeting relatively less well off. Microfinance sector is increasingly becoming regulated and they are compelling to put in place the corporate governance mechanism. Since majority of MFIs irrespective of their status are socially oriented. Therefore, good corporate governance mechanism is more effective in enhancing social performance in particular. Progress towards human development contributes to both financial and social performance of MFIs. Interestingly we conclude that increase in globalization and domestic credit make MFIs more socially oriented. Further we conclude that inflation though undermines MFIs financial performance but at the same time increases its social performance as it compels MFI to reach to the poor. Furthermore, democratic regimes are conducive for the performance of MFIs and people perception of less corruption enhances the social performance of MFIs. We further conclude that MFIs in long run tend to be more social oriented. Furthermore, MFIs having non-profit status, credit union having those lend to the group tend to be more socially oriented. 


\section{References}

Armenda'riz, B. and Szafarz, A. (2009) On mission drift of microfinance institutions, CEB Working Papers..

Bassem, S. B. (2009). Governance and performance of microfinance institutions in Mediterranean countries. Journal of Business Economics and Management, 10:1, 31 43. Baughn, C.C., Bodie, N.L., McIntosh, J.C., (2007). Corporate social and environmental responsibility in Asian countries and other geographical regions. Corp. Soc. Responsib. Environ. Manag. 14, 189e205.

Beisland, L. A., Mersland, R., \& Randøy, T. (2014). The Association between microfinance rating scores and corporate governance: a global survey. International Review of Financia Analysis, 35, 268-280.

Black, B., Kim, W., (2012). The effect of board structure on firm value: A multiple identification strategies approach using Korean data. J. Financ. Econ. 104, 203-226.

Cull, R., Demirguc, K. A., \& Morduch, J. (2007). Financial performance and outreach: A global analysis of leading microfinance banks. The Economic Journal, 117, 107-133. Chen, H., Hao, Q., (2011). Insider trading law enforcement and gross spreads of ADR IPOs. J. Bank. Financ. 35, 1907-1917.

Christen, R. P. (2001). Commercialization and mission drift: the transformation of microfinance in Latin America. CGAP Occasional Paper No. 5.

Christen, R. P., Vogel, E., \& McKean, C. (1995). Maximizing the Outreach of Microenterprise Finance: The Emerging Lessons of Successful Programs. Consultative Group to Assist the Poor.

Coleman, K. A., \& Osei, A. K. (2008). Outreach and profitability of microfinance institutions: the role of governance. Journal of Economic Studies, 34:3, 236-248.

D'Espallier, B., Goedecke, J., Hudon, M., \& Mersland, R. (2016). From NGOs to banks: Does institutional transformation alter the business model of microfinance institutions? World Development.

Denis, K. D. (2001). Twenty-five years of corporate governance research and counting.

Financial Economics, 10, 191-212.

De Jong, A., Gispert, C., Kabir, R., \& Renneboog, L. (2002). International corporate governance and firm performance: An empirical analysis. Second Draft, May. Downloadable from selene. uab. es/dep-economiaempresa/Regroup/WP_Int_Corp_Gov. pdf.

Duru, A., Iyengar, R. J., \& Zampelli, E. M. (2016). The dynamic relationship between CEO dual and firm performance: The moderating role of board independence. Journal of Business Research.

Engelen, A., Flatten, T. C., Thalmann, J., \& Brettel, M. (2014). The effect of organizational culture on entrepreneurial orientation: A comparison between Germany and Thailand. Journal of Small Business Management, 52(4), 732-752.

Fooladi, M., \& Nikzad Chaleshtori, G. (2011). Corporate governance and firm performance. In International Conference on Sociality and Economics Development (ICSED2011),Kuala Lumpur, Malaysia, June (pp. 17-19).

Ford, C. W., Nonis, S. A., \& Hudson, G. I. (2005). A cross-cultural comparison of value systems and consumer ethics. Cross Cultural Management, 12(4), 36

Gebremichael, B. Z., \& Gessesse, H. T. (2016). Technical efficiency of microfinance institutions (MFIs): does ownership matter? Evidence from African MFIs. International Journal of Development Issues, 15(3).

Glaeser, E., La Porta, R., Lopez-de-Silanes, F., Shleifer, A., 2004. Do institutions cause growth? J. Econ. Growth 9, 271-303.

Galema, R., Lensink, R., \& Mersland, R. (2012). Do Powerful CEOs Determine

Microfinance Performance? Journal of Management Studies 49:4, 718-742. 
Hahn, R., Kühnen, M., 2013. Determinants of sustainability reporting: a review of results,trends, theory, and opportunities in an expanding field of research. J. Clean. Prod. 59, 5e21.

Hartarska, V., \& Mersland, R. (2012). Which Governance Mechanisms Promote Efficiency in Reaching Poor Clients? Evidence from Rated Microfinance Institutions. European Financial Management, 18:2, 218-239.

Herrera-Echeverri, H., Geleilate, J. G., Gaitan-Riaño, S., Haar, J., \& Soto-Echeverry, N. (2016). Export behavior and board independence in Colombian family firms: The reverse causality relationship. Journal of Business Research, 69(6), 2018-2029.

Hartarska, V., \& Nadolnyak, D. (2007). Do regulated microfinance institutions achieve better sustainability and outreach? Cross-country evidence. Applied Economics, 39:10, 12071222. Howell, L. D. 2001. The Handbook of Country and Institutional Risk Analysis. East Syracuse, NY: The PRS Group.

Hurley, D., Hurley, D., Choudhary, A., \& Choudhary, A. (2016). Factors influencing attainment of CEO position for women. Gender in Management: An International Journal, 31(4), 250-265.

Im, J., \& Sun, S. L. (2015). Profits and outreach to the poor: The institutional of microfinance institutions. Asia Pacific Journal of Management,32(1), 95-117.

Janda, K., \& Turbat, B. (2013). Determinants of the financial performance of microfinance institutions in Central Asia. Post-Communist Economies, 25(4), 557-568.

Jensen, C. M. (1993). The Modern Industrial jū, Exit, and the Failure of Internal Control Systems. The Journal of Finance, 48, 831-880.

Jansson, T., Rosales, R., \& Glenn, W. D. (2004). Principles and Practices for Regulating and Supervising Microfinance. Inter-American Development Bank.

Keefer, P., (2008). Beyond legal origin and checks and balances: institutional credibility, citizen information, and financial sector devel-opment. In: Haber, S., North, D., Weingast, B (Eds.), Institutional Institutions and Financial Development. Stanford UniversityPress, Stanford, CA, pp. 125-155.

Kaufmann, D., Kraay, A., \&Mastruzzi, M. (2011). The worldwide governance indicators: Methodology and analytical issues, Hague. Journal on the Rule of Law, 3: 220-46.

Labie, M. (2001). Corporate governance in microfinance organizations: a long and winding road. Management Decision, 39:4, 296-301.

Langlois, C., \& Schlegelmilch, B. (1990). Do corporate codes of ethics reflect national character? Journal of International Business Studies, 21(4), 519-540.

Lauer, K. (2008). Transforming NGO MFIs: Critical Ownership Issues to Consider Consultative Group to Assist the Poor, Occasional-Paper, 13.

Li, J., Li, P., \& Wang, B. (2016). Do cross-border acquisitions create value? Evidence from overseas acquisitions by Chinese firms. International Business Review, 25(2), 471-483.

Ledgerwood, J., \& White, W. (2006). Transforming Microfinance institutions: Providing Full Financial Services to the Poor. The International Bank for Reconstruction and Development /The World Bank.

Labie, M. (2001). Corporate governance in microfinance organizations: a long and winding road. Management Decision, 39:4, 296-301.

Liu, Y., Miletkov, M. K., Wei, Z., \& Yang, T. (2015). Board independence and firm performance in China. Journal of Corporate Finance, 30, 223-244.

Maignan, I., Ralston, D.A., 2002. Corporate social responsibility in Europe and the US: insights from businesses' self-presentations. J. Int. Bus. Stud. 33, 497e514.

Mustafa, A. K., \& Saat, M. M. (2012). Assessing Performance And Intervention of Microfinance institutions: A Case Study In Sudan. Interdisciplinary journal of Contemporary Research In Business, 401-418.

Malik, T., \& Zhao, Y. (2013). Cultural distance and its implication for the duration of the 
international alliance in a high technology sector. International Business Review, 22(4), $699-712$.

Manderlier, A., BACQ, S., GIACOMIN, O., \& Janssen, F. (2009). The impact of South Asian Microfinance institutions corporate governance mechanisms on their social and financial performance: an exploratory study. CRECIS Center for Research in Entrepreneurial Change \& Innovative Strategies, (pp. 1-30).

Mersland R , Randøy T , Strøm $\varnothing$.(2011). The impact of international influence on microbanks performance: A global survey. International Business Review 20 (2): 163176 .

Mersland, R., \& Strom, O. (2009). Performance and governance in microfinance institutions. Journal of Banking \& Finance, 33, 662-669.

Mersland, R., \& Strom, R. O. (2008). Performance and trade-offs in anmicrofinance organizations:Does ownership matter? Journal of International Development, 20, 598 612.

Mersland, R., \& Strom, R. O. (Oct, 2013). Governance in microfinance institutions. Working Paper, University of Agder.

Microfinance Banana Skins. (2008). Microfinance Banana Skins: Risk in a booming industry. Centre for the Study of Financial Innovation (CSFI).

Microfinance Banana Skins. (2012). Microfinance Banana Skins: the CSFI survey of microfinance risk. Centre for the Study of Financial Innovation (CSFI).

Mitton, T. (2002). A cross-firm analysis of the impact of corporate governance on the East Asian financial crisis. Journal of financial economics, 64(2), 215-241.

Mori, N., Golesorkhi, S., Randøy, T., \& Hermes, N. (2015). Board composition and outreach performance of microfinance institutions: evidence from East Africa. Strategic Change, 4(1), 99-113.

Nawaz A., Iqbal S, Ehsan S. (2018). Does Social Performance Derive Corporate Governance mechanism. In case of Asian MFI? An issue of endogenity Global business review 19(4) 2018.

Périlleux, A., \& Szafarz, A. (2015). Women leaders and social performance: evidence from financial cooperatives in Senegal. World Development, 74, 437-452.

Piotroski, J.D., Wong, T.J., 2010. In: Fan, J., Morck, R. (Eds.), Institutions and Information Environment of Chinese Listed Firms.University of Chicago Press, Capitalizing China.

Polanco, O. F. (2005). Commercializing Microfinance and Deepening Outreach? Empirical Evidence from Latin America . Journal of microfinance, 7, 4769.

Qi, Y., Roth, L., Wald, J.K.,( 2010). Institutional rights and the cost of debt. J. Financ. Econ.95, 202- 226.

Rajan, R.G., Zingales, L., (2003). The great reversals: the politics of financial development in the twentieth century. J. Financ. Econ.69, 5-50

Ramady, M. A. (2014). Institutional, Economic, and Financial Country Risk: Analysis of the Gulf Cooperation Council. Heidelberg, Switzerland: Springer International.

Rao, K., \& Tilt, C. (2015, November). Board Diversity and CSR Reporting: Australian Evidence. In A-CSEAR 2015-Proceedings of the 14th Australasian Centre on Social and Environmental Accounting Research Conference: A-CSEAR 2015 (p. 116). Academic Conferences and publishing limited.

Reichert, P. (2016). A meta-analysis examining the nature of trade-offs in microfinance. Working Papers CEB, 16.

Rock, R., Otero, M., Saltzman, \& Sonia. (1998). Principles and Practices of Microfinance Governance. Microenterprise Best Practices.

Roe, M.J., Siegel, J.I., 2009. Finance and politics: a review essay based on Kenneth Dam's analysis of legal traditions in The Law-Growth Nexus. J. Econ. Lit. 473, 781- 800.

Sales, M., \& Mirvis, P. (1984). When cultures collide: Issues in acquisitions. In Kimberly \& 
Quinn (Eds.), Managing organizational translations. Homewood, IL: Irwin.

Salustri, A., \& Viganò, F. (2015). Matching profit and non-profit needs: how NPOs and cooperatives contribute to growth in time of crisis: A quantitative approach.

Schiehll, E., \& Martins, H. C. (2016). Cross-National Governance Research: A Systematic

Review and Assessment. Corporate Governance: An International Review, 24(3), 181-199

Schneider, S., \& Meyer, A. (1991). Interpreting and responding to strategic issues: The impact of national culture. Strategic Management Journal, 12(4), 307-320

Simga-Mugan, C., Daly, B. A., Onkal, D., \& Kavut, L. (2005). The influence of nationality and gender on ethical sensitivity: An application of the issue-contingent model. Journal of Business Ethics, 57, 139-159.

Srnka, K. J. (2004). Culture's role in marketers' ethical decision making: An integrated theoretical framework. Academy of Marketing Science Review, 2004, 1.

Strese, S., Adams, D. R., Flatten, T. C., \& Brettel, M. (2016). Corporate culture and absorptive capacity: The moderating role of national culture dimensions on innovation management. International Business Review, 25(5), 1149-1168.

Strøm, R. Ø., D’Espallier, B., \& Mersland, R. (2014). Female leadership, performance, and governance in microfinance institutions. Journal of Banking \& Finance, 42, 60-75.

Strøm, R. Ø., D'Espallier, B., \& Mersland, R. (2016). Female Leaders and Financial

Inclusion. Evidence from Microfinance Institutions.

Tchuigoua, T. H. (2010). Is there a difference in performance by the legal status of microfinance institutions? The Quarterly Review of Economics and Finance, 50, 436442.

The World Bank. (December 2006). Microfinance in south Asia: Towards financial inclusion for the poor. The World Bank.

Thrikawala, S., Locke, S., \& Reddy, K. (2013). Corporate Governance - Performance Relationship in Microfinance Institutions (MFIs). Asian Journal of Finance \& Accounting, 5:1, 160-182.

Thrikawala, S., Locke, S., \& Reddy, K. (2017). Dynamic Endogeneity Corporate GovernancePerformance Relationship in Microfinance sector (MFIs). Journal of Economic studies.

Tremblay, M. S., Gendron, Y., \& Malsch, B. (2016). Gender on board: deconstructing the"legitimate" female director. Accounting, Auditing \& Accountability Journal, 29(1), 165-190.

Tang, J. (2016). CEO duality and firm performance: The moderating roles of other executives and blockholding outside directors. European Management Journal.

Trompenaars, F., \& Hampden-Turner, C. (1998). Riding the waves of culture. New York: McGraw-Hill.

Van Damme, P., Wijesiri, M., \& Meoli, M. (2016). Governance and Efficiency of

MicrofinanceInstitutions Empirical Evidence from Sri Lanka. South Asia Economic Journal, 1391561416650102.

Varhegyi, M. M., \& Jepsen, D. M. (2016). Director succession planning in not-for-profit boards. Asia Pacific Journal of Human Resources.

Vanroose, A. (2007). Is microfinance an ethical way to provide financial services to the poor? Microfinance: Are its promises ethically justified? Center Emile Bernheim (CEB) Solvey Business School Working Paper No 07/014.

Vanroose, A., \& D' Espallier, B. (2013). Do microfinance institutions accomplish their mission?Evidence from the relationship between traditional financial sector development and microfinance institutions' outreach and performance. Applied Economics, 45:15, 1965-1982. Vitell, S. J., \& Paolillo, J. G. P. (2004). A crosscultural study of the antecedents of the perceived role of ethics and social responsibility. Business Ethics: A European Review, 23(April/July), 185-199

Walthoff-Borm, X., \& De Beelde, I. (2015). CEO duality and apprentice successions: 
exploringthe role of leadership structure in impression management. In 38th EAA Annual Congress.

Welbourne, T. M. (1999). Wall Street Likes its Women: An Examination of Women in the Top Management Teams of Initial Public Offerings. Cornell University ILR School CAHRS Working Paper No. 99-07.

Welford, R., 2004. Corporate social responsibility in Europe and Asia: critical elements and best practice. J. Corp. Citiz. 13, 31e47.

Welford, R., 2005. Corporate social responsibility in Europe, North America and Asia. J. Corp. Citiz. 17, 33e52.

Woller, G. (2007). Trade-offs between social and financial performance. Journal of microfinance, 9, 14-19.

Whipple, T. W., \& Swords, D. F. (1992). Business ethics judgments: A cross-cultural comparison. Journal of Business Ethics, 11(Sep), 671-678.

Zagorchev, A., \& Gao, L. (2015). Corporate governance and performance of financial institutions. Journal of Economics and Business, 82, 17-41.

Zohra, B., \& Pandey, L. S. (2011). Comparison of performance of microfinance institutions with commercial banks in india.Australian journal of Business and Management Research, 1:6, 110-120 